\title{
Drying of Gelidium sesquipedale (Rhodophyta) Under Microwave Irradiation: Study of Thermal and Kinetic Aspects of the Drying Process
}

\author{
Moulay Chrif Hnini ${ }^{1}$, M'barek Benchanaa ${ }^{1} \&$ Mustapha El Hammioui ${ }^{1}$ \\ ${ }^{1}$ Faculté des Sciences Semlalia Marrakech, Université Cadi Ayyad, Marrakech, Morocco \\ Correspondence: M'barek Benchanaa, Laboratoire de Recherche sur la Réactivité des Matériaux et \\ l'Optimisation des Procédés-REMATOP, Faculté des Sciences Semlalia Marrakech, Université Cadi Ayyad, \\ Boulevard Prince My Abdellah, B. P. 2390-40000 Marrakech, Marocco. Tel: 212-6-6119-2039. E-mail: \\ mbarek_benchanaa@yahoo.fr
}

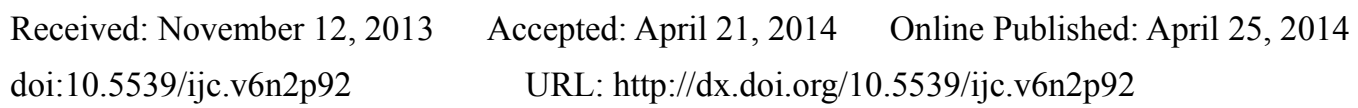

\begin{abstract}
Microwave technology is currently very interesting because it offers, in many industrial processes, a new alternative energy for drying and treatment of various materials. In this context, the drying of the Gelidium sesquipedale (Rhodophyta), which is a red algae, is carried out under microwave irradiation in the aim to optimize the energy consumption in the drying process and the quality of the extracted products. The drying process of Gelidium sesquipedale under microwave irradiation is carried out by studying the thermal and kinetic aspects of drying under different irradiation conditions. The experiments were performed on samples of different initial masses ranging from 2 to $30 \mathrm{~g}$. The samples are irradiated in an experimental device combining a gravimetric balance and microwave assembly that may impose different power microwave ranging from 50 to $200 \mathrm{~W}$.

The analysis of the experimental curves of the drying process shows that the initial mass of the sample has little influence and the applied microwave power has a significant effect on the drying kinetics. The comparative study of the drying of Gelidium sesquipedale by conventional heating and under electromagnetic field shows that the controlling kinetic mechanism depends on the way in which the sample is dried. The action of the electromagnetic field allows faster heat transfer leading to the rapid evacuation of water molecules from the seaweed.
\end{abstract}

Keywords: Gelidium sesquipedale, drying, microwave irradiation, kinetic, controlled process, energy optimization

\section{Introduction}

The drying process becomes a basic step but necessary in the food industry where the interest resides primarily in its ability to ensure a long-term storage for the food if the package is suitable. Indeed, many degradation reactions can be significantly slowed and many others can be virtually stopped (Labuza, 1975; Morris, 2011; Saravacos \& Maroulis, 2011) due to a decrease in the moisture content in the food product to values less than $25 \%$.

Over the years, the drying is performed by various methods: by convection of preheated air (Karathanos \& Belessiotis, 1997; Toğrul \& Pehlivan, 2004) direct solar drying (Yaldiz \& Ertekin, 2001; Doymaz, 2005; Vijaya Venkata Raman et al., 2012), drying by irradiation (Rodríguez et al., 2005; Wang, 2006; Zhang et al., 2006; Bilbao-Sàinz et al., 2006; Giri \& Prasad, 2007), by freeze-drying (Graciela et al., 1993; Burgschweiger \& Tsotsas, 2002; Kettner et al., 2006), under fluidized bed (Soponronnarit et al., 1997; Kannan \& Subramanian, 1998; Syahrul et al., 2002), by osmotic dehydration (Raoult-Wack, 1994; Simal et al., 1997; Della Rosa \& Giroux, 2001; Ozen et al., 2002; Mišljenović et al., 2012), etc. Currently, through continuous improvement, the thermal drying is one of the industrial operations that consume the most energy and the dehydration process is significant in the worldwide energy industry. In Morocco, an example of developing country, the energy used in the drying process is estimated at about $15 \%$ of the industrial energy consumption. This bill is about $25 \%$ in industrialized countries. This explains why our country has established a policy that encourages the promotion of 
renewable energies and new forms of energy. Morocco has substantial natural deposits of red seaweed and is the third largest producer of agar-agar. The agar-agar extracted from the Gelidium sesquipedale and its main molecule "agarose" show a very high demand in the world market. Then, the drying of this red algae becomes an indisputable necessity, because it can offer a good alternative to deal with different problems. However, to be competitive, we must seek ways of drying in the aim to prepare an agar-agar and an agarose of high organoleptic quality and well adapted to international standards. The traditional method of exposing red algae in direct sunlight cannot be a solution because it leads to an excessive loss of vitamins, due to the photo-oxidation (Bolin $\&$ Stafford, 1974), and there is a risk of contamination and insect infestation.

In a previous study (Hnini et al., 2013), we have presented the experimental results concerning the thermodynamic equilibrium of the sorption and desorption between water and the Gelidium sesquipedale. In a second work (Hnini et al., 2014), the drying of Gelidium sesquipedale was realized by conventional heating on samples with different initial masses. The influence of the drying temperature was studied by varying the temperature from 30 to $80{ }^{\circ} \mathrm{C}$. The experimental results showed that the kinetic regime that governs this transformation depends on the imposed temperature. At low temperature, the drying process is governed by an interfacial progression regime in cylindrical symmetry, and at high temperature, it well controlled by a three-dimensional diffusion.

In the present work, the drying of the Gelidium sesquipedale is performed by applying microwave irradiation which offers a high promising technology in the drying process of food. In fact, this work is inserted in an extensive program of research and development in the field of energy control by the use of microwave radiations. Drying of materials with high free water content, under microwave field, allows to understand the heating of these materials under microwave field and accordingly to optimize the energy consumption in their processes and get dry products with better quality.

The objectives of the drying of Gelidium sesquipedale under microwave irradiation are, firstly, to optimize and understand the action of the electromagnetic field on the red algae, and secondly, to achieve high yields of molecules extracted (agar-agar and agarose) from microwave-dried algae. The action of the microwave field on Gelidium sesquipedale was directed by the apprehension of the heating under microwave field and the interaction of electromagnetic field with the red alga studied as well as the behavior of the water molecule in the algae during microwave irradiation. This behavior is elucidated by comparing the different results to those obtained by conventional heating.

\section{Materials, Experimental Techniques and Operative Protocols}

The samples used in the drying study of Gelidium sesquipedale under microwave irradiation have the same characteristics as those used in the drying by conventional heating (Hnini et al., 2014). They were stored under the same conditions of temperature and humidity.

\subsection{Experimental Techniques and Operative Protocols}

As shown in the diagram in Figure 1, the experimental setup consists of a microwave assembly associated with a balance (Mettler Toledo AB104-S) which has an accuracy of $0.1 \mathrm{mg}$.

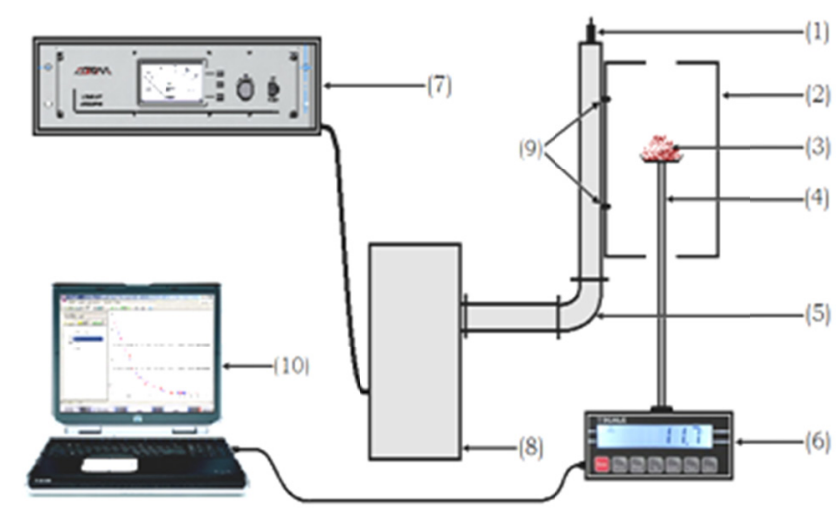

Figure 1. Experimental device used during the drying under the microwave field: (1) Connecting piston, (2) Microwave cavity, (3) Sample, (4) Glass support, (5) Waveguide, (6) Balance, (7) Player power, (8) microwave generator, (9) Radiating antennas, (10) Computer 
The microwave circuit consists of a microwave generator (8) with a maximum incident power $1500 \mathrm{~W}$ operating at $2450 \mathrm{MHz}$. This generator feeds supplies a waveguide RG 112U (5) which is closed at the other end by a connecting piston (1). The microwave energy is transmitted to a microwave cavity (2), made in steel sheet of 2 mm thick, via two radiating antennas (9).

The drying experiments are carried out under atmospheric pressure, at different incident microwave powers ranging from 50 to $200 \mathrm{~W}$ and for different initial masses of the sample ( 2 to $30 \mathrm{~g})$. The initial mass of the sample is maintained under microwave irradiation in a glass sample holder (4). The evolution of the instantaneous mass of the sample is carried out using the balance (6) and is recorded via software installed in a computer (10). The experiment is terminated when the total mass of the sample remains unchanged. The reproducibility of the results is ensured by repeating the experiment three times. The temporal evolution of the temperature is determined using a thermocouple placed inside the irradiated sample. During thermal measurements, which take place during a very short time, we proceed by stopping the microwave irradiation, for the highest incident microwave powers $(>150 \mathrm{~W})$, to avoid interaction of the electromagnetic field with thermocouple. Monitoring the evolution of the sample mass against the time is performed under constant incident microwave power and the experiments are completed when the sample mass becomes unchanged.

\section{Experimental Results}

\subsection{Kinetic Aspect of the Drying Process of the Gelidium sesquipedale}

Figure 2 shows the evolution of the mass of water desorbed $m_{d}(t)$ versus time for an initial sample mass $\mathrm{m}_{0}=10 \mathrm{~g}$ under different incident microwave powers.

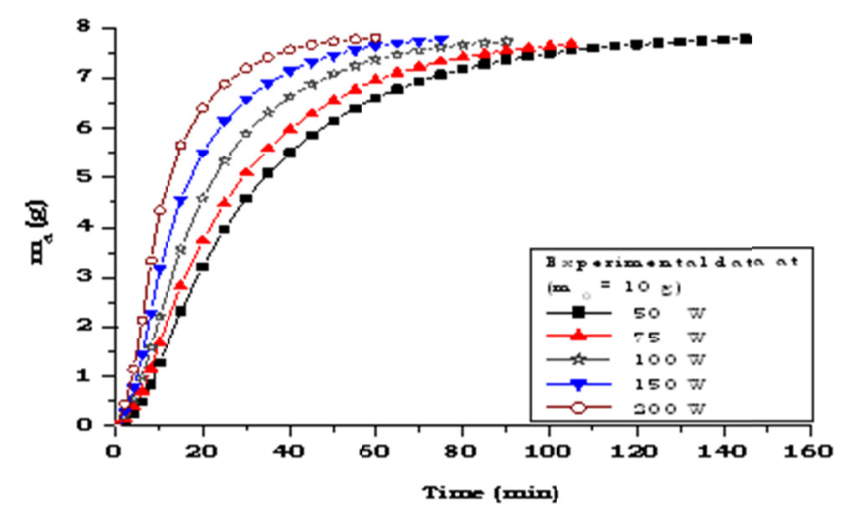

Figure 2. Influence of the microwave incident power on the drying curves of a sample of Gelidium sesquipedale of initial mass $\mathrm{m}_{0}=10 \mathrm{~g}$

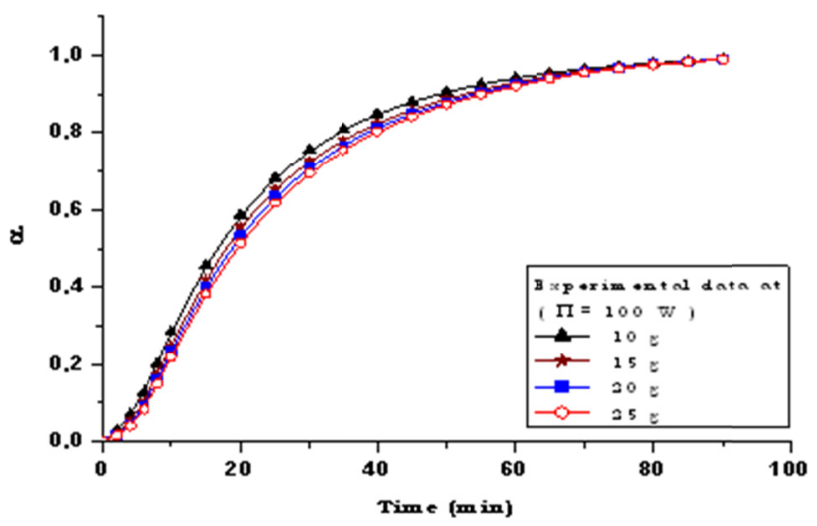

Figure 3. Effect of the initial sample mass $\mathrm{m}_{0}$ on the rate of progress during the drying of Gelidium sesquipedale under an incident microwave power of $100 \mathrm{~W}$ 
The analysis of the experimental curves obtained shows that all the curves obtained converge to the same final mass corresponding to the total desorption of the sample. For the initial mass of $10 \mathrm{~g}$ of Gelidium sesquipedale, the amount of desorbed water reaches a value close to $7.8 \mathrm{~g}(78 \%)$. The drying time decreases when the incident microwave power increase.

The desorption curves have a nearly sigmoid shape and start after a short latent period whose duration becomes shorter when the incident microwave power increases. Figure 3 illustrates the variation of the rate of progress of drying as a function of time for initial masses ranging from 10 to $25 \mathrm{~g}$. The rate progress $\alpha$ is defined as the ratio of the mass of water desorbed at time $t$ and that desorbed at the end of drying (Hnini et al., 2014):

$$
\alpha=\frac{m_{d}(t)}{m_{d}(\infty)} \quad 0 \leq \alpha \leq 1
$$

The analysis of these results shows that all the experimental curves have the same shape for all initial masses used. The drying kinetics depends slightly on the mass $\mathrm{m}_{0}$ used.

As shown in Figure 4, the desorption of water from the seaweed starts upon application of the microwave irradiation. The instantaneous rate of desorption, which is initially very low, increases gradually to reach a maximum, then decreases continuously towards zero. It is strongly influenced by the incident microwave power. The maximum speed of drying increases with the increase of the incident microwave power. In the range of the initial mass of the sample studied, the speed curves present the same evolution and show that $\mathrm{m}_{0}$ has very low effect on the kinetics of drying,

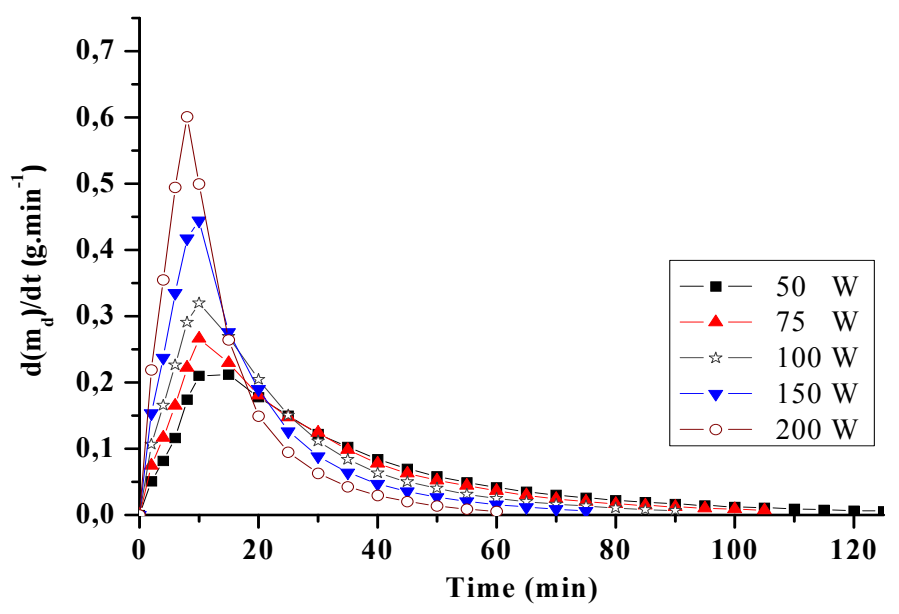

Figure 4. Drying of Gelidium sesquipedale under microwave irradiation. Variation of the instantaneous speed of drying against the time (Initial sample mass $\mathrm{m}_{0}=10 \mathrm{~g}$ )

\subsection{Mechanisms of the Drying Process Under Microwave Field}

The evolution of temperature versus time at constant incident microwave power is shown in Figure 5. The plotted curves $\theta(\mathrm{t})$ show that for every incident power:

- the maximum temperature $\theta_{\mathrm{m}}$ is as greater as the incident power is high,

- the heating is accelerated with the increasing of the incident microwave power and stopped after a time $t_{p}\left(t_{p}\right.$ $=15 \min$ for $50 \mathrm{~W}$ and $\mathrm{t}_{\mathrm{p}}=8 \mathrm{~min}$ for $200 \mathrm{~W}$ ).

- beyond this period, the drying continues at a constant temperature and the system is considered isothermal.

Taking into account the kinetic curves obtained experimentally and their evolution as a function of the incident microwave power, we note that the drying of Gelidium sesquipedale under microwave radiation seems to be realized in two different thermal phases. A first non-isothermal phase, which takes place during the transitional period $t_{p}$, is due to the heating rate which increases with the temperature imposed by the microwave field. It is followed by a second phase which is carried out under isothermal conditions. 


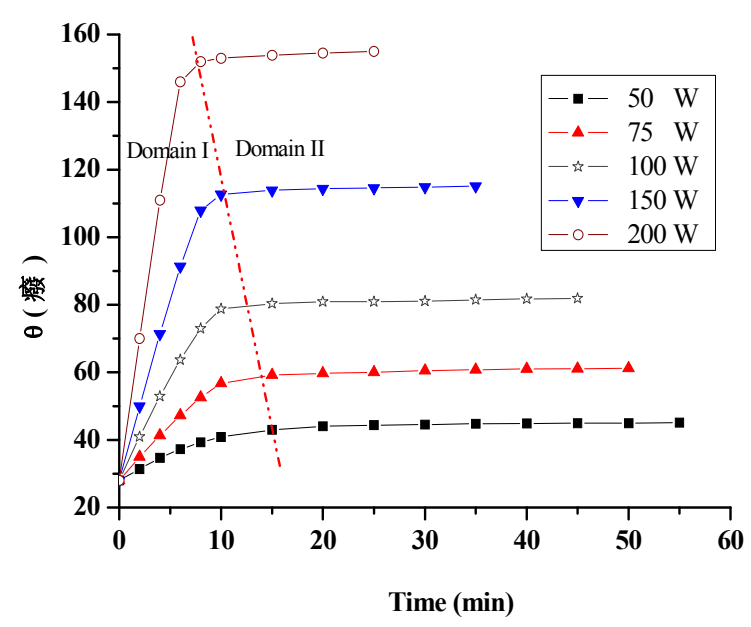

Figure 5. Thermal aspect under microwave irradiation of a sample of Gelidium sesquipedale. Curves of temperature changes for a sample of initial mass $\mathrm{m}_{0}=10 \mathrm{~g}$

As described in our previous work (Hnini et al., 2014), the variation of rate of progress $\alpha$ with time follows a differential law of the form:

$$
\frac{d \alpha}{d t}=k(T) \cdot f(\alpha)
$$

which leads by integration, under isothermal conditions to the function $F(\alpha)$, given by:

$$
F(\alpha)=\int_{0}^{\alpha} \frac{d \alpha}{f(\alpha)}=\int_{0}^{t} k(T) d t=k(T) \bullet t
$$

For a non-isothermal process, Equation (2) leads to the Achar equation (Achar et al., 1966), which is often used to describe the experimental curves obtained under non-isothermal conditions (Coats \& Redfern, 1963, 1964; Jarez et al., 1987):

$$
\operatorname{Ln}\left(\frac{\frac{d \alpha}{d t}}{f(\alpha)}\right)=\operatorname{Ln}\left(\frac{A}{\beta}\right)-\frac{E_{a}}{R} \cdot \frac{1}{T}
$$

where $\beta=(\mathrm{dT} / \mathrm{dt})$ is the heating rate, $A$ is the pre-exponential factor and $E_{a}$ the apparent activation energy.

To determine the kinetic regime that controls the drying process under microwave irradiation, we use kinetic functions which are widely used in heterogeneous kinetic processes for the determination of isothermal and non-isothermal reaction mechanisms (Hnini et al., 2014). Indeed, two approaches were discussed to describe the shapes of the experimental curves. The first approach is to check the linearity of the first part of the curves, where the drying is performed with a non-isothermal heating rate which depends on the incident microwave power. The use of Equation (4) provides a better linearization if we choose $f(\alpha)=1 / 2 \alpha$ which reflects a drying process controlled by a monodimensional diffusion regime comparable to that of the evaporation of weakly bounded water molecules. For each incident microwave power, the apparent activation energy $E_{a}$ can be determined from the plotted $\operatorname{Ln}\left(\frac{d \alpha}{d t} / f(\alpha)\right)$ versus 1/T (Figure 6). The values of the apparent activation energy are reported in Table 1. 


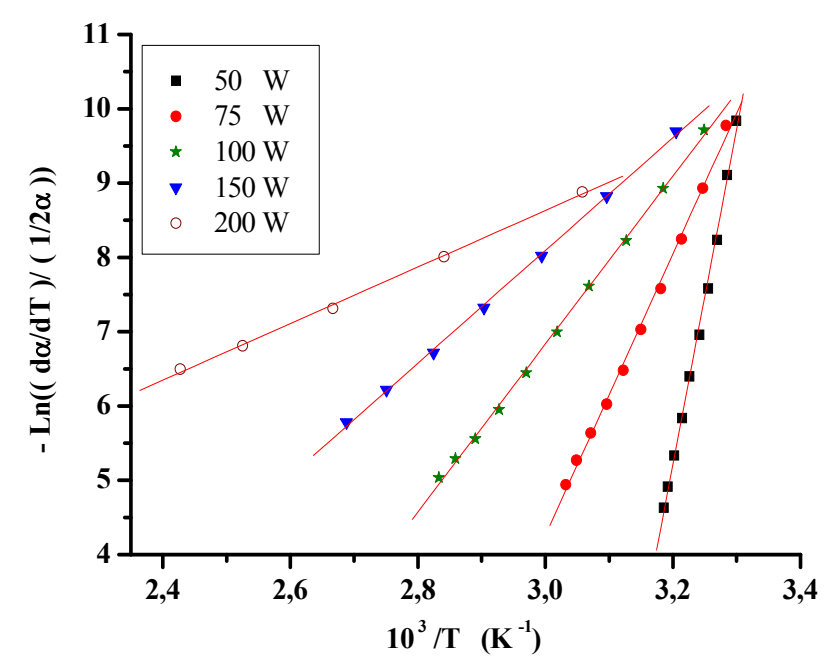

Figure 6. Variation of $\operatorname{Ln}\left(\frac{d \alpha}{d t} / f(\alpha)\right)$ versus $1 /$ T according the Achar equation (Equation (4))

Table 1. Drying of Gelidium sesquipedale under microwave irradiation. Values of the activation energy $\mathrm{E}_{\mathrm{a}}$ (given by the Achar's method)

\begin{tabular}{ccc}
\hline$\pi(\mathrm{W})$ & $\beta\left({ }^{\circ} \mathrm{C} \cdot \mathrm{min}^{-1}\right)$ & $\mathrm{E}_{\mathrm{a}}\left(\mathrm{kJ} \cdot \mathrm{mol}^{-1}\right)$ \\
\hline 50 & 1.4 & 89.2 \\
75 & 3.5 & 37.6 \\
100 & 5.5 & 22.4 \\
150 & 10.5 & 15.1 \\
200 & 22.5 & 7.6 \\
\hline
\end{tabular}

The second approach allows to the linearization of the second part of the curves $\left(t>t_{p}\right)$ using the isothermal kinetic models characterized by the kinetic equations, depending in particular on the nature of the mechanism of the phenomenon (decomposition, dehydration, drying). The experimental curves are better transformed (Figure 7) according to the Jander's equation reported by Sharp et al. (1966):

$$
F(\alpha)=\left[1-(1-\alpha)^{1 / 3}\right]^{2}=k^{\prime}(T)\left(t-t_{p}\right)
$$

which shows that beyond of $t_{p}$, the kinetic regime that regulates the drying process is the three-dimensional diffusion.

The slopes $\mathrm{k}^{\prime}(\mathrm{T})$ are attributed to the rate constants and related to the temperature imposed by the microwave field. According to the Arrhenius law, the plotted of Lnk'(T) versus 1/T (Figure 8) is a straight line that allows the determination of the apparent activation energy $E_{\mathrm{a}}$. The value obtained is $10.3 \mathrm{~kJ} \cdot \mathrm{mol}^{-1}$. 


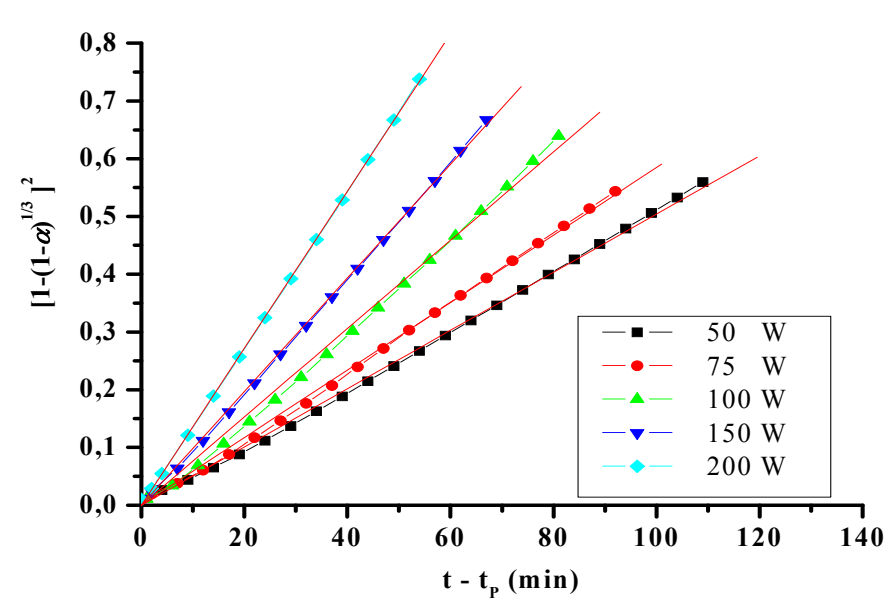

Figure 7. Linear transformations of the drying curves under different incident powers

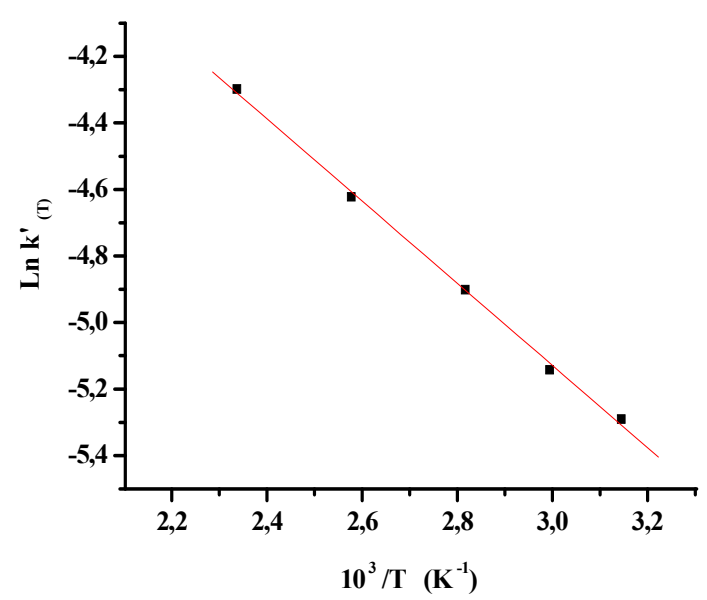

Figure 8. Variation of Lnk'(T) versus $1 / \mathrm{T}$ according to a tri-dimensional diffusion in spherical symmetry

\section{Discussion and Interpretation of Results}

The analysis of all results provides information on the kinetics and mechanisms of the thermal drying and confirms the nature of water molecules previously defined (Hnini et al., 2013). The kinetic mechanism that regulates the drying process under microwave field is not unique (Figure 8, domains I and II). Two mechanisms of desorption of water molecules have been identified in this work:

- A mono-dimensional diffusion mechanism, similar to that obtained during a rapid evaporation of molecules of free water surface, takes place at the beginning of the drying process, after a time corresponding to the setting temperature of the sample under microwave irradiation (Figure 5, domain I). If the intensity of the microwave field is high, its effect on the weakly bound water molecules is important, and the amount of desorbed water is high. The drying process studied here concerns the desorption of $80 \%$ of water weakly bound molecules. It should be noted that the apparent activation energy is close to the heat obtained during the evaporation of free water, which is about $43.9 \mathrm{~kJ} \cdot \mathrm{mol}^{-1}$. The apparent activation energy, determined from the slope of the transformed curves, encompasses the true value of the activation energy of drying process and other values related to the thermodynamics quantities.

- A mechanism of three-dimensional diffusion of water molecules according to the Jander's law, which takes place beyond the first minutes, once the temperature reaches the thermal level of the applied microwave power (Figure 5, Domain II). This heat level, similar to a thermal equilibrium, can be linked to a transient state caused by the balance of the microwave energy desorbed (heating mode) and the energy needed for desorption of water molecules (endothermic phenomenon). The heat transfer to the heart of the sample is 
quick and the desorption of water molecules is also very fast. The drying is done quickly with a mass transfer influenced by the effect of the imposed temperature. The global phenomenon is controlled by the three-dimensional diffusion characterized by the linearization of the experimental curves according to the Jander's equation. This diffusive regime is sometimes dominated by mechanical phenomena which start from the first minutes.

The results also indicate that the apparent activation energy depends on the temperature imposed by the microwave power and how the heat is transferred to the algae. The value of the apparent activation energy obtained under these conditions (domain II) is about $10.3 \mathrm{~kJ} \cdot \mathrm{mol}^{-1}$. This difference in the apparent activation energy is due, on the one hand, unlike the mechanisms involved, and secondly, by the mechanical phenomena that begin early in domain I.

\section{Conclusion}

In this work, we studied the kinetic and thermal aspects of drying of Gelidium sesquipedale under microwave irradiation for different initial masses. Two main objectives were set: understanding the influences of the incident microwave power and the initial mass of the sample and determining the mechanisms that control the drying and kinetic parameters that can be used to optimize the drying.

The experimental results were obtained by performing drying under atmospheric pressure at different incident microwave powers varying from 50 to $200 \mathrm{~W}$ for different initial masses of between 10 and $25 \mathrm{~g}$. The evolution of the instantaneous mass $m_{d}(t)$ of the sample is carried out by gravimetry using a balance. The experience is completed when the total mass of the sample remains unchanged and the reproducibility of the results is ensured by repeating the experiment three times.

The temporal evolution of the temperature within the sample under different microwave powers shows the existence of two areas: a first one is characterized by a linear increase in temperature with a drying speed proportional to the microwave power used, followed by a second of which operates isothermally. During the first area, we have applied the Achar method which is a non-isothermal kinetic model. The linearization of the curves obtained allowed to determine values of the activation energy ranging between 7.5 and $89.2 \mathrm{~kJ} \cdot \mathrm{mol}^{-1}$. In the second area the kinetic of the isothermal regime is a three-dimensional diffusion according to the Jander's equation; the corresponding activation energy is about $10.3 \mathrm{~kJ} \cdot \mathrm{mol}^{-1}$.

The comparison of these results with those obtained during drying by conventional thermally conducted at atmospheric pressure at different temperatures ranging from 30 to $80{ }^{\circ} \mathrm{C}$ with different initial sample masses (Hnini et al., 2014) shows that:

- The drying time by conventional heating is greater than the drying time under microwave irradiation which corresponds to a gain in energy.

- During the conventional heating, the initial sample mass has a significant effect on the kinetic as well as on the duration of the drying process. This will result in more energy consumption at industrial level when drying large masses (overcrowding effect) unlike the microwave technology where the mass effect is small because the microwave irradiation reacts inside the material.

- The dried sample by microwave maintains its color and its original form whereas for the conventional way, rods become distorted and take a darker color particularly to the high temperatures. This can be explained by the degradation of organic matter in the seaweed. A further work is being finalized, focuses on the qualitative and quantitative of the amount of extracted agar-agar and agarose from the sesquipedale Gelidium dried by conventionnel heating and under microwave irradiation.

\section{References}

Berteli, M. N., Rodier, E., \& Marsaioli, A. (2009). Study of the microwave vacuum drying process for a granulated product. Brazilian Journal of Chemical Engineering, 26(2), 317-329. http://dx.doi.org/10.1590/S0104-66322009000200009

Bilbao-Sàinz, C, Andrés, A., Chiralt, A., \& Fito, P. (2006). Microwaves phenomena during drying of apple cylinders. Journal of Food Engineering, 74(1), 160-167. http://dx.doi.org/10.1016/j.jfoodeng.2005.02.028

Bolin, H. R., \& Stafford, A. E. (1974). Effect of processing and storage on provitamin A and vitamine C in apricot. Journal of Food Science, 39(5), 1034-1036. http://dx.doi.org/10.1111/j.1365-2621.1974.tb07305.x

Burgschweiger, J., \& Tsotsas, E. (2002). Experimental investigation and modelling of continuous fluidized bed drying under steady-state and dynamic conditions, Chemical Engineering Science, 57(24), 5021-5038. http://dx.doi.org/10.1016/S0009-2509(02)00424-4 
Coats, A. W., \& Redfern, J. P. (1963). Thermogravimetric analysis. Analyst, 88, 906-924. http://dx.doi.org/10.1039/AN9638800906

Coats, A. W., \& Redfern, J. P. (1964). Kinetic parameters from thermogravimetric. Nature, 201, 68-69. http://dx.doi.org/10.1038/201068a0

Della Rosa, M., \& Giroux, F. (2001). Osmotic Treatments (OT) and Problems Related to the Solution $\begin{array}{llll}\text { Management, Journal of Food } & \text { Engineering, } 23-236 .\end{array}$ http://dx.doi.org/10.1016/S0260-8774(00)00216-8

Doymaz, I. (2005). Sun drying of figs: An experimental study. Journal of Food Engineering, 71(4), 403-407. http://dx.doi.org/10.1016/j.jfoodeng.2004.11.003

Giri, S. K., \& Prasad, S. (2007). Drying kinetics and rehydration characteristics of microwave-vacuum and convective hot-air dried mushrooms. Journal of Food Engineering, 78(2), 512-521. http://dx.doi.org/10.1016/j.jfoodeng.2005.10.021

Graciela, F., De Valdez, G. F., \& Diekmann, H. (1993) Freeze drying conditions of starter cultures for sourdoughs. Cryobiology, 30(2), 185-190. http://dx.doi.org/10.1006/cryo.1993.1018

Hnini, M. C., Benchanaa, M., \& El Hammioui, M. (2013). Study of the interaction between water and Gelidium sesquipedale (Rhodophyta). Part I: Thermodynamic aspect of the sorption equilibrium. Journal of the Taiwan Institute of Chemical Engineers, 44(5), 795-801. http://dx.doi.org/10.1016/j.jtice.2013.01.023

Hnini, M. C., Benchanaa, M., \& El Hammioui, M. (2014). Study of the interaction between water and Gelidium sesquipedale (Rhodophyta). Part II - Kinetic of the drying process. Accepted in the International Journal of Chemistry.

Jander, W. (1927). Reactions in the solid state at high temperature. Zeitschrift fur anorganische und allgemeine Chemie, 163(1), 1-30. http://dx.doi.org/10.1002/zaac.19271630102

Jarez, A., Ramos, E., Gaitán, M., Veiga, M. L., \& Pico, C. (1987). A method for the kinetic analysis of non-isothermal decomposition reactions of solids. Thermochimica Acta, 115, 175-180. http://dx.doi.org/10.1016/0040-6031(87)88363-6

Kannan, C. S., \& Subramanian, N. B. (1998). Some drying aspects of multistage fluidized beds. Chemical Engineering Technololgy, 21(12), 961-966. http://dx.doi.org/10.1002/(SICI)1521-4125(199812)21:12<961::AID-CEAT961>3.0.CO;2-0

Karathanos, V. T., \& Belessiotis, V. G. (1997). Sun and artificial air drying kinetics of some agricultural products. Journal of Food Engineering, 31(1), 35-46. http://dx.doi.org/10.1016/S0260-8774(96)00050-7

Kettner, C., Peglow, M., Metzger, T., \& Tsotsas, E. (2006). Distributed product in fluidized bed drying. In Proceedings of the $X V^{\text {th }}$ International Drying Symposium, Budapest, Vol. B, pp. 745-750.

Labuza, T. P. (1975). Sorption phenomena in foods: Theoretical and Practical Aspects. In Chokyun Rha (Ed.), Theory, Determination and Control of physical properties of food materials, Series in Food Material Science, 1, 197-219. http://dx.doi.org/10.1007/978-94-010-1731-2_11

Mišljenović, N. M., Koprivica, G. B., Pezo, L. L., Lević, L. B., Ćurčić, B. Lj., Filipović, V. S., \& Nićetin, M. R. (2012). Optimization of the osmotic dehydration of carrot cubes in sugar beet molasses. Thermal Science, 16(1), 43-52. http://dx.doi.org/10.2298/TSCI110808129M

Morris, S. A. (2011). Food and package Engineering. John Wiley \& Sons Inc.

Ozen, B. F., Dock, L. L., Ozdemir, M., \& Floros, J. D. (2002). Processing factors affecting the osmotic dehydration of diced green peppers. International Journal of Food Science and Technology, 37(5), 497-502. http://dx.doi.org/10.1046/j.1365-2621.2002.00606.x

Raoult-Wack, A. L. (1994). Resent advances in the osmotic dehydration of foods. Trends in Food Science and Technology, 5(8), 255-260. http://dx.doi.org/10.1016/0924-2244(94)90018-3

Rodríguez, R., Lombraña, J. I., Kamel, M., \& de Elvira, C. (2005). Kinetic and quality study of mushroom drying under microwave and vacuum. Drying Technology, 23(9-11), 2197-2213. http://dx.doi.org/10.1080/07373930500212685

Roura, P., \& Farja, J. (2009). Analytical solution for the Kissinger equation. Journal of Materials Research, 24(10), 3095-3098. http://dx.doi.org/10.1557/jmr.2009.0366

Saravacos, G. D., \& Maroulis, Z. B. (2011). Food Process Engineering Operations. CRC press, Taylor and 
Francis Group, New York.

Sharp, J. H., Brindley, G. W., \& Narahari Achar, B. N. (1966). Numerical data for some commonly used solid state reaction equations. Journal of American Ceramic Society, 49(7), 379-382. http://dx.doi.org/10.1111/j.1151-2916.1966.tb13289.x

Simal, S., Deyá, E., Frau, M., \& Rosselló, C. (1997). Simple modelling of air drying curves of fresh and osmotically pre-dehydrated apple cubes. Journal of Food Engineering, 33(1-2), 139-150. http://dx.doi.org/10.1016/S0260-8774(97)00049-6

Soponronnarit, S., Pongtornkulpanich, A., \& Prachayawarakorn, S. (1997). Drying characteristics of corn in fluidized bed dryer. Drying Technology, 15(5), 1603-1615. http://dx.doi.org/10.1080/07373939708917311

Syahrul, S., Hamdullahpur, F., \& Dincer, I. (2002). Energy analysis in fluidized-bed drying of large wet particles. International Journal of Energy Research, 26(6), 507-525. http://dx.doi.org/10.1002/er.799

Toğrul, I. T., \& Pehlivan, D. (2004). Modelling of thin layer drying kinetics of some fruits under open-air sun drying process. Journal of Food Engineering, 65(3), 413-425. http://dx.doi.org/10.1016/j.jfoodeng.2004.02.001

Vijaya Venkata Raman, S., Iniyan, S., \& Goic, R. (2012). A review of solar drying technologies. Renewable and Sustainable Energy Reviews, 16, 2652-2670. http://dx.doi.org/10.1016/j.rser.2012.01.007

Wang, J., \& Sheng, K. (2006). Far-infrared and microwave drying of peach. LWT-Food Science and Technology, 39(3), 247-255. http://dx.doi.org/10.1016/j.lwt.2005.02.001

Yaldiz, O., \& Ertekin, C. (2001). Thin layer solar drying of some different vegetables. Drying Technology - An International Journal, 19(3-4), 583-597. http://dx.doi.org/10.1081/DRT-100103936

Zhang, M., Tang, J., Mujumadar, A. S., \& Wang, S. (2006). Trends in microwave related drying of fruits and

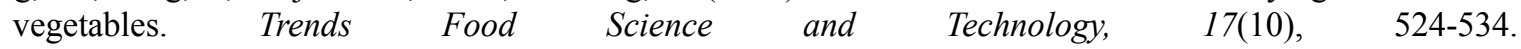
http://dx.doi.org/10.1016/j.tifs.2006.04.011

\section{Copyrights}

Copyright for this article is retained by the author(s), with first publication rights granted to the journal.

This is an open-access article distributed under the terms and conditions of the Creative Commons Attribution license (http://creativecommons.org/licenses/by/3.0/). 